1 Universidade Federal do Paraná (UFPR) - Curitiba (PR), Brasil.

prof.rafaelgd@gmail.com

2 Universidade Federal do Paraná (UFPR) - Curitiba (PR), Brasil.

thabata.cristy@hotmail.com

3 Pontifícia Universidade Católica do Paraná (PUCPR) - Curitiba (PR), Brasil simone.moyses@pucpr.br

4 Pontifícia Universidade Católica do Paraná (PUCPR) - Curitiba (PR), Brasil. s.moyses@pucpr.br

\section{A contratualização como ferramenta da gestão na Atenção Primária à Saúde na percepção dos profissionais da Secretaria Municipal de Saúde de Curitiba, Brasil}

Contracting as a management tool of Primary Health Care in the perception of professionals of the Municipal Health Department of Curitiba, Brazil

Rafael Gomes Ditterich', Thabata Cristy Zermiani2 ${ }^{\mathbf{2}}$ Simone Tetu Moysés ${ }^{\mathbf{3}}$, Samuel Jorge Moysés $\mathbf{4}$

RESUMO Objetivou-se analisar o uso da contratualização como ferramenta da gestão na Atenção Primária à Saúde no município de Curitiba. A metodologia consistiu na análise dos dados secundários, produzidos pelo Banco Mundial em pesquisa realizada em 2005, referentes à gestão dos cuidados primários à saúde. A contratualização foi considerada importante na organização do processo de trabalho, sendo útil para a identificação e solução de problemas, para o estabelecimento de prioridades e de compromissos mútuos entre os profissionais, os gestores e a população. No entanto, há ainda lacunas na efetivação da gestão pactuada e no processo de negociação das metas.

PALAVRAS-CHAVE Contratos; Atenção Primária à Saúde; Gestão em saúde; Negociação.

ABTRACT This article aims to analyze the use of contracting as a tool for the management of Primary Health Care in the county of Curitiba. The method used was the analysis of secondary data, produced by the World Bank on research conducted in 2005, regarding the management of primary health care. The contracting was considered important in the organization of the work process, being useful for identifying and solving problems, to establishing priorities, and mutual commitments between professionals, managers and the public. However, there are still gaps in the fulfillment of the agreed management and in the process of negotiation of goals.

KEYWORDS Contracts; Primary Health Care; Health management; Negotiation. 


\section{Introdução}

A contratualização em saúde é um tema importante nas agendas de discussão em âmbito nacional e internacional (DITTERICH; MOYSÉS; MOYSÉS, 2012). Vários países, tanto de orientação bismarckiana como beveridgiana adotaram diferentes processos de contratualização a fim de propiciar maior efetividade dos cuidados à saúde e maior eficiência no uso dos recursos. Como exemplos, podem ser citados a Austrália, o Canadá, a Espanha, o Reino Unido, a Holanda, a Alemanha e a França (ESCOVAL; RIBEIRO; MATOS, 2010). Portugal já propunha mudanças em seu processo organizacional e a negociação de metas desde 1996. No entanto, foi a partir de 2005 , com o projeto de reconfiguração dos centros de saúde e implementação das unidades de saúde familiares em Portugal que os mecanismos de contratualização foram de fato instituídos (NEY; PIERANTONI; LAPÃO, 2015).

No Brasil, sobretudo após o ano de 1990, a implantação do Sistema Único de Saúde (SUS) impôs a busca por novas formas de gestão em saúde. As instâncias deliberativas/executivas passaram por um processo de descentralização e as decisões foram deslocadas para os níveis locais. Integrando o repertório dos mecanismos de gestão em saúde, algumas cidades e estados no Brasil implantaram o processo de contratualização, o qual tem sido empregado tanto no setor hospitalar como na atenção primária. Também tem sido uma ferramenta bastante utilizada nas parcerias com o terceiro setor, como verificado entre as Organizações Sociais (OS) e o governo na gestão de diversos hospitais, sendo que, atualmente, passaram também a ser celebrados com a administração direta, nas pactuações internas em secretarias e órgãos dos governos municipais e estaduais (DITTERICH; MOYSÉS; MOYSÉS, 2012). Nesse sentido, ressalta-se o entendimento explicitado na Portaria $\mathrm{n}^{\circ} 4.279$, de 30 de dezembro de 2010 (BRASIL, 2010), segundo o qual é necessária a criação de mecanismos formais de contratualização que abranjam todos os pontos da Rede de Atenção à Saúde a fim de que se tenha uma gestão eficaz, eficiente e qualificada.

Em estudos de Costa e Silva (2014) são apresentados quatro dispositivos de contratualização no Brasil: o contrato interno estabelecido entre uma Secretaria Municipal de Saúde (SMS), unidades de saúde e equipes pertencentes à administração direta, com destaque ao município de Curitiba (PR), que o instituiu de forma inovadora em 2002, e Belo Horizonte, em 2011; o contrato de gestão entre a Fundação Estatal de Saúde da Família (Fesf-SUS) na Bahia, vinculado à administração indireta e os munícipios deste estado a ela associados; o contrato de gestão estabelecido entre Secretarias de Saúde e Organizações Sociais, como aqueles implantados no estado de São Paulo e do Rio de Janeiro; e o contrato interno entre munícipios e governo federal na esfera do Programa Nacional de Melhoria do Acesso e da Qualidade da Atenção Básica (PMAQ-AB) instituído em 2011.

Em todas as situações discriminadas, o processo de contratualização tem por objetivo propiciar melhorias na saúde da população, atendendo de forma efetiva as suas necessidades; obter um controle sobre as despesas de origem pública com a saúde, alcançando maior eficiência na gestão dos recursos; coordenar as ações das partes envolvidas, assegurando que os compromissos sejam cumpridos; além de disponibilizar informação aos cidadãos (BRASIL, 2010).

Nesse contexto, pode ser definida como um processo de relacionamento entre financiadores e prestadores, fundamentado em uma filosofia contratual, em que há uma clara ligação entre o financiamento atribuído e os resultados esperados. Esse processo se fundamenta na autonomia e responsabilidade das partes e na existência de um sistema de informação que possibilite o planejamento e a avaliação de forma 
eficaz, sendo objetos de contrato as metas de produção, acessibilidade, qualidade e eficiência (FREITAS, 2002 APUD ESCOVAL, 2003). Araújo (2010) ressalta que, para a efetivação desse processo, devem ser estabelecidas metas, que orientem a ação da gestão; meios, que criem condições para o alcance das metas; controle, permitindo o acompanhamento do processo e da alocação de recursos e a avaliação da implantação das ações; e os incentivos, que mobilizam o comportamento humano em direção aos objetivos propostos.

O documento assinado pelas partes que viabiliza essa forma de atuação estatal pode receber várias denominações, como contratos de gestão ou termos de compromissos. Ressalta-se, ainda, que eles podem ser classificados como contratos endógenos ou exógenos. Os primeiros são aqueles em que o 'contratante' e o 'contratado' são instituições/entidades estatais, como os contratos que secretarias municipais de saúde estabelecem com suas unidades de prestação de serviços ou com unidades administrativas desconcentradas (COSTA E SILVA, 2004). Por outro lado, os contratos exógenos são aqueles em que o ente contratante é uma instituição estatal que se relaciona com entes prestadores externos, como instituições públicas não estatais, instituições privadas filantrópicas ou lucrativas (MENDES, 2002).

Escoval, Ribeiro e Matos (2010), ao analisar o processo de contratualização na Atenção Primária à Saúde (APS) em diversos países, constataram que se trata de um instrumento necessário para o alcance de maior eficiência, acesso mais amplo aos serviços de saúde, prestação de cuidados com maior qualidade, com enfoque na prevenção e na promoção da saúde. Entretanto, ainda há algumas falhas, entre elas, a falta de orientações claras e adequadas por parte do governo, a dificuldade em conciliar metas predefinidas ao orçamento da saúde, a desarticulação entre orçamento e execução financeira, a falta de um programa de reestruturação organizacional e a capacitação insuficiente dos profissionais envolvidos (ESCOVAL, 2010).

Freitas e Escoval (2010) apontam a necessidade de blindar o sistema à volatilidade do sistema político, uma vez que o ciclo de vida político é efêmero para a produção de efeitos das reformas estruturais. Nesse sentido, esses contratos devem instituir mecanismos de participação social, propiciando a transparência do processo e seu monitoramento contínuo, desde o planejamento fundamentado nas necessidades dos cidadãos até o financiamento justo da atividade em questão. Costa e Silva, Escoval e Hortale (2014) afirmam ser necessária uma pactuação bilateral, permitindo que os grupos anteriormente considerados como objetos da ação gerencial atuem como sujeitos de formulação de diretrizes.

Em Curitiba, no estado do Paraná, a partir do seu processo de municipalização, a Secretaria Municipal da Saúde (SMS Curitiba) optou por redesenhar sua política de saúde, bem como a gestão desta política, passando do controle vertical centralizado em instâncias superiores, para a gestão, monitoramento e avaliação de resultados, com base em negociações políticas. A primeira e principal tarefa realizada foi o planejamento e a programação das ações de saúde, em relações horizontalizadas entre população e equipe de saúde, em nível local, em um processo ascendente para o nível distrital e nível central. Nesse processo de discussão, a SMS criou o cargo de Autoridade Sanitária Local (ASL), como forma de melhor gerir a atenção primária, sendo a ASL responsável pela coordenação e chefia da Unidade Básica de Saúde (UBS) e da saúde da população na sua área de abrangência (DUCCI, 2007).

Em 2002, construiu-se a proposta de desenvolvimento da contratualização na atenção primária, tendo por objetivo o aumento da participação, negociação e responsabilidade mútua entre os gestores, as equipes de saúde e a comunidade. Posteriormente, em 2003, essa proposta 
foi implantada em todas as UBS e Distritos Sanitários. No período compreendido entre 2002 e 2007, o aprimoramento deste instrumento de gestão, o qual foi denominado Termo de Compromissos (Tercom), aliado ao sistema de remuneração variada e ferramentas de planejamento, possibilitou a consolidação do sistema de avaliação participativa, voltado para resultados (AZEVEDO; FAORO; XAVIER, 2013).

O Tercom, além do aspecto administrativo, também traz um componente ético e moral, uma vez que não dispõe de uma base legal para a cobrança dos compromissos assumidos (MENDES, 2002). Esses Termos de Compromissos, em âmbito municipal, são firmados anualmente entre a equipe de saúde, o gestor da unidade de saúde e o gestor distrital, culminando com o gestor municipal, que deve responder à população em última instância. Neste momento, verifica-se também a participação de representantes dos usuários, membros dos Conselhos Locais de Saúde. Têm como elementos constitutivos básicos os objetivos e metas fixados nos instrumentos básicos de planejamento, como o Plano Municipal de Saúde, a Programação Anual de Saúde, o Pacto pela Saúde, o Planejamento Local, entre outros, visando coordenar e garantir que estes compromissos sejam cumpridos (AZEVEDO; FAORO; XAVIER, 2013).

Ao assinar o Tercom, as unidades de saúde e os Distritos Sanitários formalizam a organização do processo de trabalho, buscando identificar e resolver problemas, estabelecendo prioridades e incentivando relações intersetoriais com outras áreas da administração municipal (DUCCl, 2007). Um dos componentes do Tercom é o Plano Operativo Anual (POA), o qual consiste em uma planilha, composta por indicadores, metas pactuadas para cada nível institucional, bem como fontes para monitoramento (AZEVEDO; FAORO; XAVIER, 2013). O POA possui 74 indicadores, que são monitorados trimestralmente, possibilitando que as equipes acompanhem e observem os seus resultados (AZEVEDO, 2009). Existem metas pactuadas para o nível local (unidades de Atenção Básica) e outras específicas para os Distritos Sanitários.

A contratualização, além da assinatura do Tercom, contempla mecanismos de monitoramento e avaliação e um sistema de incentivos, chamado Programa de Incentivo ao Desenvolvimento da Qualidade (IDQ), propondo o pagamento de incentivos financeiros mensais, por meio de gratificações sobre o salário-base, aos funcionários que apresentarem bons resultados em seu desempenho (DucCl, 2007). Esse programa encontra-se vinculado à busca de resultados por meio do desempenho do indivíduo e da equipe, e sua aplicação sustenta-se em um sistema de avaliação articulado com os indicadores de resultados contratados na SMS por meio dos contratos de gestão. O IDQ é avaliado trimestralmente por uma equipe de gestão de recursos humanos da SMS Curitiba por meio de quatro planilhas: avaliação individual do profissional da saúde (realizada pela chefia), autoavaliação, avaliação da unidade de saúde e avaliação da comunidade (AZEVEDO; FAORO; XAVIER, 2013).

A análise do processo de contratualização em nível local é de grande valia na medida em que é neste nível que ocorre a prestação de serviços, bem como a pactuação e negociação das metas. A escolha do município de Curitiba para realização desta análise se justifica pelo fato de ser uma das experiências pioneiras no âmbito da administração pública e da APS no Brasil, desenvolvida desde 2003, o que revela a maturidade desta política. A importância deste tema é evidenciada pela expansão dessa ferramenta de gestão, em suas diferentes modalidades, em vários municípios e estados nas diferentes modalidades de gestão.

Baseando-se nos argumentos apresentados, o presente artigo tem como objetivo analisar o uso do Tercom como ferramenta da gestão na APS no município de Curitiba, Brasil. 


\section{Métodos}

Este estudo fundamentou-se na análise dos dados secundários produzidos em 2005 pelo Banco Mundial, o qual pesquisou inovações em gestão no Brasil, incluindo a gestão hospitalar em São Paulo e a gestão dos cuidados primários à saúde no município de Curitiba. No ano de 2006, o Banco Mundial publicou o relatório técnico 'Brazil enhancing performance in Brazil's Health Sector: lessons from innovations in the state of São Paulo and the city of Curitiba', no qual apresentou os dados e informações de forma geral e objetiva. No entanto, a sua base de dados somente se tornou pública após o período de cinco anos.

A pesquisa do Banco Mundial realizou um levantamento da percepção dos servidores que atuavam em suas unidades de saúde sobre o uso e aplicabilidade do contrato interno de gestão (Tercom). Ela foi enviada, por meio do sistema de correio interno da SMS, para 372 funcionários elegíveis (nível superior) em uma amostra representativa de unidades de saúde do município. $\mathrm{O}$ total de participantes que responderam ao questionário foi de 254 servidores (a taxa de resposta foi de 68\%). As 31 unidades participantes da amostra foram escolhidas para fornecer uma representação adequada de todos os Distritos de Saúde, bem como a distribuição por tipo de cargos e carreiras superiores constituintes da equipe mínima da atenção primária no município (ASL, médico, enfermeiro e cirurgião-dentista), e da distribuição entre unidades básicas com Equipes de Saúde da Família e unidades básicas sem Equipes de Saúde da Família (WORLD BANK, 2006). As questões do instrumento de coleta de dados de pesquisa foram propostas, após discussão com a direção da SMS, tendo em vista que o principal objetivo das entrevistas de campo seria compreender se o sistema de gestão por resultados, com a pactuação de metas, teria potencial para influenciar o desempenho institucional global. O questionário foi composto de 29 questões de múltipla escolha referentes ao Tercom, ao
Conselho Local de Saúde, à influência política e ao IDQ. A pesquisa do Banco Mundial restringiu-se à avaliação das frequências das respostas sobre o ponto de vista do total de participantes, realizando somente a análise descritiva dos dados, sem considerar a análise mais detalhada dos diferentes atores envolvidos no processo.

Este artigo faz parte de uma pesquisa maior intitulada 'A contratualização como ferramenta de gestão e organização da Atenção Primária à Saúde: avanços, dificuldades e desafios', desenvolvida em 2011, a qual objetivou analisar a percepção dos trabalhadores da saúde no município de Curitiba, acerca do Tercom e do IDQ como ferramentas da gestão e organização na APS no município. Para sua realização, foi obtido termo de consentimento da SMS para a cessão e uso da base de dados secundários gerados com a pesquisa do Banco Mundial, citada anteriormente. O protocolo de pesquisa apresentado como pré-requisito à execução deste trabalho foi submetido à análise pelo Comitê de Ética em Pesquisa da Pontifícia Universidade Católica do Paraná, tendo sido devidamente aprovado sob $\mathrm{n}^{\circ} 5667$ e parecer $\mathrm{n}^{\circ}$ 004066/10, em 30 de junho de 2010.

Esta pesquisa utilizou a base de dados secundária produzida pelo Banco Mundial, realizando um trabalho prévio de filtragem e consistência dos dados, excluindo-se da análise as ASL e os profissionais que apresentavam menos de um ano atuando nas unidades de saúde, ao tempo em que as entrevistas foram realizadas. Considerou-se o critério de que para responder aos itens pesquisados os participantes deveriam já ter se apropriado do sistema de avaliação do desempenho, com um mínimo de tempo de serviço. Dessa forma, entre os 254 servidores que responderam ao questionário do Banco Mundial, foram utilizadas nesta pesquisa as respostas de 196 deles, entre os quais se tornaram elegíveis aos critérios de inclusão: 25 ASL, 71 médicos, 30 enfermeiros e 70 cirurgiões-dentistas. Houve 
pequena variação do número de participantes (perdas amostrais) dependendo da pergunta em análise, pois elas eram variáveis independentes, existindo a possibilidade de se abster de responder ao item. Das 29 questões aplicadas pelo Banco Mundial, na presente análise foram selecionadas 3 para este artigo referentes ao uso do Tercom para a organização do processo de trabalho, para a identificação e solução de problemas, para estabelecimento de prioridades e para a avaliação da negociação sobre metas pactuadas.

Outro importante ponto de diferenciação entre os resultados apresentados no relatório do Banco Mundial e a presente pesquisa foi a preocupação desta em avaliar a percepção das diferentes categorias profissionais sobre o Tercom. O recorte metodológico proposto buscou identificar a existência de diferenças na percepção de cada categoria profissional (médicos, enfermeiros e cirurgiões-dentistas) ante a percepção da ASL, sobre a utilização das metas e indicadores pactuados na organização da APS em Curitiba. A análise dos dados foi realizada pelo software SPSS ${ }^{\circledast}$ for Windows v. 13.0. O teste de U Mann-Whitnney, com nível de significância de 5\%, foi aplicado para verificar a diferença entre as opiniões das ASL comparado com os outros cargos profissionais participantes sobre os contratos internos de gestão.

\section{Resultados}

Ao analisar as respostas obtidas nos questionários, observou-se na base dados secundários que em algumas perguntas houve abstenções de poucos profissionais referentes ao item avaliado, o que foi permitido na pesquisa do Banco Mundial. Isso justifica a diferença de respondentes nas distintas categorias avaliadas em cada uma das tabelas do trabalho (tabela 1 a 5), o que não compromete a análise, já que para cada pergunta foi realizada uma análise estatística. As perguntas, apesar de avaliarem o Tercom, não eram variáveis dependentes.

A avaliação do Tercom enquanto ferramenta na organização do processo de trabalho nas unidades de saúde no município revelou que este se constitui em um instrumento substancialmente ou totalmente importante para a maioria dos profissionais, atingindo um percentual próximo a $80 \%$ em cada categoria profissional (tabela 1). Contudo, ao comparar as opiniões das ASL com a dos profissionais da equipe básica de saúde, constataram-se divergências na distribuição de respostas, já que os médicos (17,6\%) e cirurgiões-dentistas $(23,2 \%)$ apontam pouco ou parcial efeito sobre a organização do processo de trabalho, ao passo que este percentual foi de apenas $10 \%$ para os enfermeiros e de $0 \%$ para as ASL.

Tabela 1. Avaliação do Tercom como ferramenta na organização do processo de trabalho pelos diferentes profissionais participantes no município de Curitiba (PR), 2005

\begin{tabular}{|c|c|c|c|c|c|c|}
\hline \multirow[t]{2}{*}{ Cargo } & \multicolumn{4}{|c|}{ O Tercom é útil para a organização do processo de trabalho } & \multirow[t]{2}{*}{ Total } & \multirow{2}{*}{$\begin{array}{c}\text { Teste U Mann- Whitney } \\
\text { ( p ) }\end{array}$} \\
\hline & Minimamente & Parcialmente & Substancialmente & Totalmente & & \\
\hline \multicolumn{7}{|l|}{ Autoridade } \\
\hline Sanitária & 0 & 0 & $8(32,0 \%)$ & $17(68,0 \%)$ & $25(100 \%)$ & $0,006^{\star}$ \\
\hline Médico & $2(2,9 \%)$ & $10(14,7 \%)$ & $29(42,7 \%)$ & $27(39,7 \%)$ & $68(100 \%)$ & \\
\hline \multicolumn{7}{|l|}{ Autoridade } \\
\hline Sanitária & 0 & 0 & $8(32,0 \%)$ & $17(68,0 \%)$ & $25(100 \%)$ & 0,281 \\
\hline Enfermeiro & 0 & $3(10,0 \%)$ & $10(33,3 \%)$ & $17(56,7 \%)$ & $30(100 \%)$ & \\
\hline \multicolumn{7}{|l|}{ Autoridade } \\
\hline Sanitária & 0 & 0 & $8(32,0 \%)$ & $17(68,0 \%)$ & $25(100 \%)$ & $0,024^{\star}$ \\
\hline Cirurgião-dentista & $2(2,9 \%)$ & $14(20,3 \%)$ & $20(29,0 \%)$ & $33(47,8 \%)$ & $69(100 \%)$ & \\
\hline
\end{tabular}

Fonte: Dados do World Bank (2006).

Nota: * Estatisticamente significante $(p \leq 0,05)$ 
Ao analisar a utilidade do Tercom na identificação e solução dos problemas nas unidades de saúde (tabela 2), os servidores participantes sinalizaram que ele é uma ferramenta substancialmente ou totalmente útil para este aspecto, com um percentual que variou de $65 \%$ a $91 \%$ para as diferentes categorias profissionais. Percebeu-se que a maioria dos entrevistados escolheu a opção totalmente útil para ambos os aspectos, excetuando a categoria profissional dos médicos, cuja percepção quanto à utilidade para a solução de problemas foi majoritariamente substancial. Também se constatou que as ASL apresentam percepção mais positiva nesses itens do que os profissionais que atuam na atenção à saúde.

Tabela 2. Avaliação do Tercom como ferramenta para a identificação e solução de problemas pelos diferentes profissionais participantes no município de Curitiba (PR), 2005

\begin{tabular}{|c|c|c|c|c|c|c|}
\hline \multirow[t]{2}{*}{ Cargo } & \multicolumn{4}{|c|}{ O Tercom é útil para a identificação de problemas } & \multirow[t]{2}{*}{ Total } & \multirow{2}{*}{$\begin{array}{c}\text { Teste U } \\
\text { Mann- Whitney } \\
\text { (p) }\end{array}$} \\
\hline & Minimamente & Parcialmente & Substancialmente & Totalmente & & \\
\hline $\begin{array}{l}\text { Autoridade } \\
\text { Sanitária }\end{array}$ & 0 & $2(8,3 \%)$ & $7(29,2 \%)$ & $15(62,5 \%)$ & $24(100 \%)$ & 0,208 \\
\hline Médico & 0 & $12(17,9 \%)$ & $22(32,8 \%)$ & $33(49,3 \%)$ & $67(100 \%)$ & \\
\hline $\begin{array}{l}\text { Autoridade } \\
\text { Sanitária }\end{array}$ & 0 & $2(8,3 \%)$ & $7(29,2 \%)$ & $15(62,5 \%)$ & $24(100 \%)$ & 0,668 \\
\hline Enfermeiro & 0 & $3(10,0 \%)$ & $10(33,3 \%)$ & $17(56,7 \%)$ & $30(100 \%)$ & \\
\hline $\begin{array}{l}\text { Autoridade } \\
\text { Sanitária }\end{array}$ & 0 & $2(8,3 \%)$ & $7(29,2 \%)$ & $15(62,5 \%)$ & $24(100 \%)$ & 0,116 \\
\hline Cirurgião-dentista & $4(5,8 \%)$ & $10(14,5 \%)$ & $23(33,3 \%)$ & $32(46,4 \%)$ & $69(100 \%)$ & \\
\hline \multirow[t]{2}{*}{ Cargo } & \multicolumn{4}{|c|}{ O Tercom é útil para a solução de problemas } & Total & Teste U \\
\hline & Minimamente & Parcialmente & Substancialmente & Totalmente & & $\begin{array}{c}\text { Mann- Whitney } \\
\text { (p) }\end{array}$ \\
\hline $\begin{array}{l}\text { Autoridade } \\
\text { Sanitária }\end{array}$ & 0 & $4(16,7 \%)$ & $8(33,3 \%)$ & $12(50 \%)$ & $24(100 \%)$ & 0,056 \\
\hline Médico & $4(6 \%)$ & $13(19,4 \%)$ & $32(47,8 \%)$ & $18(26,9 \%)$ & $67(100 \%)$ & \\
\hline $\begin{array}{l}\text { Autoridade } \\
\text { Sanitária }\end{array}$ & 0 & $4(16,7 \%)$ & $8(33,3 \%)$ & $12(50,0 \%)$ & $24(100 \%)$ & 0,939 \\
\hline Enfermeiro & 0 & $4(13,3 \%)$ & $12(40,0 \%)$ & $14(46,7 \%)$ & $30(100 \%)$ & \\
\hline $\begin{array}{l}\text { Autoridade } \\
\text { Sanitária }\end{array}$ & 0 & $4(16,7 \%)$ & $8(33,3 \%)$ & $12(50,0 \%)$ & $24(100 \%)$ & 0,151 \\
\hline Cirurgião-dentista & $6(8,6 \%)$ & $18(25,7 \%)$ & $18(25,7 \%)$ & $28(40,0 \%)$ & $70(100 \%)$ & \\
\hline
\end{tabular}

Fonte: Dados do World Bank (2006).

A tabela 3 revela que a maioria dos profissionais identificou ser o Tercom um instrumento bastante importante para o estabelecimento de prioridades, com percentuais superiores a $80 \%$ para todas as categorias, ao considerar as classificações substancialmente e totalmente. Todavia, ao analisar a percepção discriminada entre os diferentes profissionais avaliados, os enfermeiros avaliaram mais positivamente esta questão do que as ASL, ao considerar a ferramenta com $100 \%$ substancialmente ou totalmente importante; sendo que, ao considerar apenas a classificação totalmente, nota-se que as ASL obtiveram um maior percentual (79,1\%). Já os médicos e cirurgiões-dentistas avaliaram mais negativamente esse item, sendo esta diferença de posições estatisticamente significante. 
Tabela 3. Avaliação do Tercom como ferramenta para o estabelecimento de prioridades pelos diferentes profissionais participantes no município de Curitiba (PR), 2005

\begin{tabular}{|c|c|c|c|c|c|c|}
\hline \multirow[t]{2}{*}{ Cargo } & \multicolumn{4}{|c|}{ O Tercom é útil para o estabelecimento de prioridades } & \multirow[t]{2}{*}{ Total } & \multirow{2}{*}{$\begin{array}{c}\text { Teste U } \\
\text { Mann- } \\
\text { Whitney } \\
\text { (p) }\end{array}$} \\
\hline & Minimamente & Parcialmente & Substancialmente & Totalmente & & \\
\hline $\begin{array}{l}\text { Autoridade } \\
\text { Sanitária }\end{array}$ & 0 & $1(4,2 \%)$ & $4(16,7 \%)$ & $19(79,1 \%)$ & $24(100 \%)$ & $0,030^{*}$ \\
\hline Médico & $2(3,0 \%)$ & $8(12,1 \%)$ & $20(30,3 \%)$ & $36(54,6 \%)$ & $66(100 \%)$ & \\
\hline $\begin{array}{l}\text { Autoridade } \\
\text { Sanitária }\end{array}$ & 0 & $1(4,2 \%)$ & $4(16,7 \%)$ & $19(79,1 \%)$ & $24(100 \%)$ & 0,175 \\
\hline Enfermeiro & 0 & 0 & $12(40,0 \%)$ & $18(60,0 \%)$ & $30(100 \%)$ & \\
\hline $\begin{array}{l}\text { Autoridade } \\
\text { Sanitária }\end{array}$ & 0 & $1(4,2 \%)$ & $4(16,7 \%)$ & $19(79,1 \%)$ & $24(100 \%)$ & $0,016^{*}$ \\
\hline Cirurgião-dentista & 0 & $11(15,7 \%)$ & $23(32,9 \%)$ & $36(51,4 \%)$ & $70(100 \%)$ & \\
\hline
\end{tabular}

Fonte: Dados do World Bank (2006).

Nota: * Estatisticamente significante $(p \leq 0,05)$.

Relativamente ao processo de pactuação das metas do Tercom (tabela 4), mais de $50 \%$ dos respondentes de cada classe profissional consideraram haver uma negociação parcial das metas, já que o gestor do Distrito Sanitário impõe algumas metas e renegocia outras. Entretanto, ao analisar o percentual de profissionais que relatou a imposição de metas, pode-se perceber uma variação, sendo que $15,5 \%$ dos médicos,
$13,8 \%$ dos enfermeiros e $11,6 \%$ dos cirurgiões-dentistas destacam a imposição de metas pela gestão municipal, sendo este percentual de somente $4,2 \%$ entre as ASL. Outro ponto a ser identificado, apesar do número reduzido de servidores respondentes a esta questão, é que somente as ASL e os médicos apontaram que a gestão municipal sugere algumas metas ao invés de impô-las.

Tabela 4. Avaliação do processo de negociação de metas no Tercom pelos diferentes profissionais participantes no município de Curitiba (PR), 2005

\begin{tabular}{|c|c|c|c|c|c|c|}
\hline \multirow[t]{2}{*}{ Cargo } & \multicolumn{4}{|c|}{ Sobre o processo de pactuação do Tercom com o Distrito Sanitário } & \multirow[t]{2}{*}{ Total } & \multirow{2}{*}{$\begin{array}{c}\text { Teste U } \\
\text { Mann- Whitney } \\
\text { (p) }\end{array}$} \\
\hline & Impõe metas & $\begin{array}{l}\text { Impõe metas e } \\
\text { negocia outras }\end{array}$ & Negocia metas & Sugere metas & & \\
\hline $\begin{array}{l}\text { Autoridade } \\
\text { Sanitária }\end{array}$ & $1(4,2 \%)$ & $13(54,1 \%)$ & $9(37,5 \%)$ & $1(4,2 \%)$ & $24(100 \%)$ & 0,069 \\
\hline Médico & $11(15,5 \%)$ & $42(59,2 \%)$ & $16(22,5 \%)$ & $2(2,8 \%)$ & $71(100 \%)$ & \\
\hline $\begin{array}{l}\text { Autoridade } \\
\text { Sanitária }\end{array}$ & $1(4,2 \%)$ & $13(54,1 \%)$ & $9(37,5 \%)$ & $1(4,2 \%)$ & $24(100 \%)$ & 0,234 \\
\hline Enfermeiro & $4(13,8 \%)$ & $16(55,2 \%)$ & $9(31,0 \%)$ & 0 & $29(100 \%)$ & \\
\hline $\begin{array}{l}\text { Autoridade } \\
\text { Sanitária }\end{array}$ & $1(4,2 \%)$ & $13(54,1 \%)$ & $9(37,5 \%)$ & $1(4,2 \%)$ & $24(100 \%)$ & 0,089 \\
\hline Cirurgião-dentista & $8(11,6 \%)$ & $43(62,3 \%)$ & $18(26,1 \%)$ & 0 & $69(100 \%)$ & \\
\hline
\end{tabular}

Fonte: Dados do World Bank (2006). 
$\mathrm{Na}$ eventualidade de ocorrer a situação da equipe não cumprir as metas pactuadas (tabela 5), percebeu-se que a maioria dos profissionais concorda plenamente ou parcialmente com a existência de abertura do Distrito Sanitário à renegociação, atingindo um percentual variável entre $75 \%$ e $100 \%$ entre as categorias profissionais. Observou-se que as ASL obtiveram o maior percentual de plena concordância (72\%), já os demais profissionais afirmaram que nem sempre o Distrito Sanitário está aberto à renegociação; somente em algumas situações permite-se justificar (e mudar) as metas pactuadas no Tercom. Esta diferença entre a opinião das ASL e a dos outros profissionais é estatisticamente significante, já que a distribuição das respostas é bem divergente, pois enquanto as ASL apontam somente os itens 'concordo parcialmente' e 'concordo plenamente', os outros servidores apresentam distribuição nos itens 'discordo totalmente', 'discordo parcialmente' e 'indiferente'.

Tabela 5. Avaliação no caso de não cumprimento das metas pactuadas pelos diferentes profissionais participantes do município de Curitiba (PR), 2005

\begin{tabular}{|c|c|c|c|c|c|c|c|}
\hline \multirow[b]{2}{*}{ Cargo } & \multicolumn{5}{|c|}{$\begin{array}{l}\text { No caso do não cumprimento das metas pactuadas, o Distrito Sanitário é aberto a negociá- } \\
\text { las }\end{array}$} & \multirow[b]{2}{*}{ Total } & \multirow{2}{*}{$\begin{array}{c}\text { Teste U } \\
\text { Mann- } \\
\text { Whitney } \\
\text { (p) }\end{array}$} \\
\hline & $\begin{array}{c}\text { Discordo } \\
\text { totalmente }\end{array}$ & $\begin{array}{c}\text { Discordo } \\
\text { parcialmente }\end{array}$ & Indiferente & $\begin{array}{c}\text { Concordo } \\
\text { parcialmente }\end{array}$ & $\begin{array}{c}\text { Concordo } \\
\text { plenamente }\end{array}$ & & \\
\hline $\begin{array}{l}\text { Autoridade } \\
\text { Sanitária }\end{array}$ & 0 & 0 & 0 & $7(28,0 \%)$ & $18(72,0 \%)$ & $25(100 \%)$ & $0,002^{*}$ \\
\hline Médico & $4(5,8 \%)$ & $1(1,5 \%)$ & $8(11,6 \%)$ & $29(42,0 \%)$ & $27(39,1 \%)$ & $69(100 \%)$ & \\
\hline $\begin{array}{l}\text { Autoridade } \\
\text { Sanitária }\end{array}$ & 0 & 0 & 0 & $7(28,0 \%)$ & $18(72,0 \%)$ & $25(100 \%)$ & $0,008^{*}$ \\
\hline Enfermeiro & 0 & $4(13,8 \%)$ & $3(10,3 \%)$ & $10(34,5 \%)$ & $12(41,4 \%)$ & $29(100 \%)$ & \\
\hline $\begin{array}{l}\text { Autoridade } \\
\text { Sanitária }\end{array}$ & 0 & 0 & 0 & $7(28,0 \%)$ & $18(72,0 \%)$ & $25(100 \%)$ & $0,032^{*}$ \\
\hline Cirurgião-dentista & $3(4,5 \%)$ & $2(3,0 \%)$ & $4(6,1 \%)$ & $24(36,4 \%)$ & $33(50,0 \%)$ & $66(100 \%)$ & \\
\hline
\end{tabular}

Fonte: Dados do World Bank (2006).

Nota: * Estatisticamente significante $(p \leq 0,05)$

\section{Discussão}

O contrato de gestão é um instrumento gerencial proposto, idealmente, para reduzir a distância entre a gestão estratégica e o centro operacional, isto é, entre o planejamento e a implementação das ações, conjugando, em um ambiente institucional 'pacificado' a negociação e a adoção de compromissos pactuados. Assim, a contratação pode compensar a diferenciação entre o técnico e o administrativo e a diferenciação entre categorias profissionais, reduzindo tensionamentos gerados por subjetividades, expectativas ambíguas e conflitos de autoridade - por exemplo, entre a autoridade do especialista (conhecimento) e a autoridade administrativa (hierarquia). Visa, discursivamente, o desenvolvimento de fóruns de discussão ampliada, com ênfase no trabalho em equipe (COSTA E SILVA, 2004).

No entanto, para que isso ocorra, Fortuna et al. (2002) apontam que é necessário romper com a dicotomia na organização do processo de trabalho instituída em grande parte dos municípios, em que o gestor ou uma equipe técnica é responsável pelo planejamento e avaliação das ações e programas de saúde e que os trabalhadores, na linha de frente nas UBS, são apenas responsabilizados pela execução, em uma delegação de tarefas muitas 
vezes alienante. Os resultados deste estudo evidenciaram que ainda há imposição de algumas metas no município de Curitiba. Ressalta-se, assim, a necessidade de haver corresponsabilidade e compromisso entre os trabalhadores e o nível diretivo, para que sejam atingidas as finalidades da gestão por resultados. As metas devem, portanto, ser pactuadas entre todos os atores envolvidos e avaliadas periodicamente (DITTERICH; MOYSÉS; MOYSÉs, 2012).

Sobre o sistema gerencial de saúde em Curitiba, Mendes (2007) argumenta que este possui pontos fortes, pois é sustentado por um planejamento estratégico; desenvolve-se por meio de um plano tático que deriva de uma planilha de programação contida em cada protocolo clínico; a partir destas planilhas fazem-se as programações anuais em cada unidade de saúde; estas programações geram um contrato de gestão entre a Secretaria e os profissionais; e estes contratos podem gerar um sistema de incentivos para as equipes que cumprem as metas programadas.

Uma avaliação preliminar com os servidores da saúde sobre o instrumento contratual na gestão da APS em Curitiba concluiu que o Tercom é útil para organizar os processos de trabalho, identificar e resolver problemas, esclarecer objetivos e estabelecer prioridades, entre outros benefícios (WORLD BANK, 2006; MENDES, 2007). Em consonância com tal avaliação, no presente estudo, embora tenha se observado uma variação nas respostas entre as diferentes categorias profissionais, a maioria dos respondentes também considerou este instrumento útil para a organização do processo de trabalho, para a identificação e solução de problemas e para o estabelecimento de prioridades,

A SMS de Curitiba, em documento publicado pelo Ministério da Saúde, relatou que, com a implantação dessa ferramenta, foram observadas mudanças nos processos de trabalho, gerência, planejamento e acompanhamento de indicadores. Aprendeu-se manejo clínico, estratificação de risco, protocolos, perfil epidemiológico e gestão por resultados. Verificou-se melhoria dos indicadores locais, maior compromisso das equipes, melhor distribuição das atribuições, melhor convivência, reforço das atitudes positivas, reconhecimento de objetivos comuns e o incremento da ação multiprofissional (BRASIL, 2006). Azevedo, Faoro e Xavier (2013) constataram mudanças de postura entre os profissionais, que passaram a ser ativos e orientados para a busca de resultados. Além disso, observou-se melhora no desempenho dos serviços e dos indicadores locais de saúde.

No entanto, apesar de os dados combinados para todas as categorias profissionais pesquisadas, mais as ASL, serem sugestivos de avanços em algumas dimensões do processo de trabalho com repercussões sobre a gestão/avaliação, a presente pesquisa identificou que algumas condições avaliadas sobre o Tercom não foram unanimemente percebidas e respondidas. Tal fato pode ser verificado ao analisar, por exemplo, a resposta às questões sobre a utilidade do Tercom na organização do processo de trabalho e no estabelecimento de prioridades, uma vez que a percepção dos médicos e cirurgiões-dentistas divergiu da percepção das ASL e dos enfermeiros.

Azevedo (2009), reaplicando as mesmas questões avaliadas pelo Banco Mundial, mas incluindo as categorias profissionais de nível médio, também identificou diferenças entre as respostas das ASL e as dos profissionais de saúde de nível superior e de nível médio. A autora também constatou que as melhores percepções sempre foram identificadas pelas ASL, seguidas pelos profissionais de nível superior e os de nível médio, respectivamente.

Esses dados permitem algumas outras interrogações e o diálogo com a literatura, pois, segundo Alves, Penna e Brito (2004), o enfermeiro é o profissional de saúde que historicamente tem ocupado a grande maioria dos cargos de chefia/coordenação de unidades de saúde, uma vez que o 
curso de graduação em enfermagem é um dos poucos da área da saúde que possuem conteúdos/disciplinas curricularmente voltados para a formação em administração. Ainda assim, não consegue formar um gerente com as capacidades exigidas na contemporaneidade para enfrentar as novas demandas impostas pela administração pública. Os autores consideram que, entre os servidores da saúde, o enfermeiro, por ter essa formação diferenciada, acaba se destacando em seu posicionamento no cumprimento de normas e no repasse de ideologias institucionais. Certamente, esses argumentos não são consensuais e inquestionáveis, mas há de se considerar a hipótese de que os enfermeiros estão mais habituados a processos administrativos em unidades de saúde, a rotinas e processos de normatização e regras na organização do processo de trabalho, além de apresentarem maior familiaridade na utilização de informações e indicadores de saúde no planejamento local em saúde.

Assim, é possível que enfermeiros e ASL consigam perceber mais nitidamente uma das principais finalidades do Tercom, que, segundo Azevedo (2009), visa ao aprimoramento do planejamento e avaliação local, na medida em que o monitoramento das ações é permanente, melhorando a viabilidade técnica, política e financeira das ações prestadas pela SMS à população de Curitiba. Isso pode ser percebido nas questões que analisaram se o Tercom é útil à identificação e solução de problemas, pois os profissionais afirmam que ele tem grande poder de identificação e substancial potencial para a solução dos problemas.

Observou-se uma visão mais positiva dos gestores, principalmente em relação à pactuação de metas, quando comparados aos servidores que atuam na linha de frente do cuidado. Talvez em virtude de os profissionais serem a 'face' visível no cotidiano das relações institucionais com a população, experimentado as contradições e desafios da atenção à saúde, e tendo que cumprir metas, processos e rotinas. Para Fortuna et al. (2002), na grande maioria dos municípios brasileiros, os administradores das unidades de saúde são indicados politicamente pelos gestores municipais, sem permitir aos trabalhadores da saúde a discussão e participação no processo decisório da escolha da melhor pessoa para assumir o cargo. Nota-se, assim, a necessidade de tornar mais claro o processo de definição dos gestores, analisando as competências de cada um, para que essa escolha seja definida através do mérito.

Um aspecto importante observado em algumas respostas neste estudo está relacionado à imposição de metas e à falta de negociação por parte da gestão municipal. Ayala e Oliveira (2007), ao avaliarem as tensões entre os profissionais de saúde e gestores no município de Joinville (SC), argumentam que, entre os vários problemas que conspiram contra a pactuação, destaca-se o fato do gestor operar, muitas vezes, com base em mecanismos impositivos para o alcance de metas, em detrimento das condições dos trabalhadores, frequentemente operando acima dos seus limites, comprometendo a qualidade dos serviços ofertados e sendo pouco resolutivos quanto às necessidades dos usuários.

Nesse sentido, Araújo (2010) destaca a importância da avaliação ex-post, que consiste na comparação entre as metas estabelecidas e as metas realizadas, para verificar os acertos e erros da tomada de decisão em um determinado momento, permitindo que se planeje o futuro; e que o profissional de saúde se aproprie das metas e indicadores pactuados pela gestão, participando das decisões e se sentindo corresponsável pela execução das ações.

A fim de ampliar a responsabilização sobre os resultados desejados, faz-se necessário aprimorar os mecanismos de controle e monitoramento, definir precisamente os indicadores e seus padrões no campo da assistência e da gestão e capacitar os envolvidos 
na elaboração do plano (DITTERICH; MOYSÉS; MOYś́s, 2012). Ney, Pierantoni e Lapão (2015) ressaltam alguns fatores que podem constituir pontos de fragilidade, como o processo de seleção dos indicadores de monitoramento, os quais podem não privilegiar as necessidades locais de saúde da população, podendo não representar melhoria de qualidade.

Para Ditterich, Moysés e Moysés (2012), é necessária a análise do processo de trabalho sob o ponto de vista coletivo, e não apenas na singularidade da produção individual. Talvez baseada nesses preceitos, a SMS demonstrou preocupação com essa questão, pois as metas são consideradas coletivamente, por unidade de saúde, o que demonstra um ganho em relação ao trabalho em equipe, pois é estimulado um dispositivo tal qual um "efeito sentinela", desencadeando uma preocupação em rede na busca da produção do cuidado em saúde que foi pactuado (DUCCl, 2007, P. 52).

Em relação a algumas contradições percebidas nos resultados, será importante que a gestão municipal reveja o eventual caráter impositivo de algumas metas e a forma de renegociá-las, pois os trabalhadores da saúde acabam não compreendendo o porquê de algumas metas não serem passíveis de renegociação. Para isso, faz-se necessário por parte da SMS desvelar que muitos desses indicadores e metas são pactuados primeiramente em nível federal e estadual, antes mesmo de serem discutidos e acordados em nível local. Quando os servidores da saúde souberem o que a gestão espera deles e conhecerem realmente os critérios que estão sendo pactuados, a SMS de Curitiba terá um sistema de gestão por resultados ainda mais eficaz e que tenha como objetivo principal a qualidade de seus serviços de saúde.

\section{Conclusão}

A Secretaria Municipal de Saúde de Curitiba, ao implantar mecanismos gerenciais, como o Tercom, institucionalizou a ideia de que resultados prioritários devem ser pactuados e alcançados na gestão da atenção primária. Por meio de metas e objetivos preestabelecidos no contrato interno de gestão, os quais são avaliados objetivamente e de forma contínua, o município desenvolveu mecanismos e ferramentas que ressaltam a importância da corresponsabilidade e da instituição de compromissos mútuos entre os trabalhadores da saúde em nível local, a gestão municipal e a população. Iniciativas como essa reforçam a importância da presença dos servidores da saúde no processo de definição de metas e objetivos institucionais, demonstrando ser uma maneira criativa e inovadora para enfrentar e repensar os problemas na gestão em saúde pública.

Restam os desafios de se repensar a gestão por resultados de uma forma em que o processo de trabalho seja analisado sob o ponto de vista coletivo, e não somente na singularidade da produção individual. Com base nos resultados apresentados, há ainda lacunas importantes separando a enunciação discursiva da efetivação concreta da gestão pactuada, sendo necessário que os profissionais de saúde se apropriem de todas as etapas de planejamento, elaboração, execução e avaliação das ações e programas pactuados no Tercom. Também é importante que a pactuação de resultados seja um investimento humano permanente, não somente respeitando parâmetros quantitativos e de produtividade, mas também observando centralmente que a produção do cuidado em saúde exige a humanização do trabalho, a satisfação dos usuários e a geração de impacto socioepidemiológico.

Estudos como este são relevantes na medida em que propiciam o reconhecimento dos aspectos positivos e negativos desse instrumento por aqueles que estão diretamente envolvidos com a atenção à saúde. A partir desse reconhecimento, é possível adequá-lo, corrigindo os seus aspectos falhos e ampliando sua efetividade. Além disso, os resultados 
obtidos são úteis visto que permitem a tomada de consciência de tais aspectos também por outros municípios que estão adotando os contratos de gestão de forma mais recente.

\section{Referências}

\author{
ALVES, M.; PENNA, C. M. M.; BRITO, M. J. M. Perfil \\ dos gerentes de Unidades Básicas de Saúde. Revista \\ Brasileira de Enfermagem, Brasília, DF, v. 57, n. 4, p. \\ 441-446, 2004.
}

ARAÚJO, M. A. D. Responsabilização pelo controle de resultados no Sistema Único de Saúde no Brasil. Revista Panamericana de la Salud Pública, Washington, v. 27 , n. 3 , p. $230-236,2010$.

AYALA, A. L. M.; OLIVEIRA, W. F. A divisão do trabalho no setor da saúde e a relação social de tensão entre trabalhadores e gestores. Revista Trabalho, Educação e Saúde, Rio de Janeiro, v. 5, n. 2, p. 217-241, 2007.

AZEVEDO, J. C. R. Contrato interno de gestão e plano operacional anual da secretaria municipal da saúde de Curitiba, Paraná: a percepção dos gestores locais e trabalhadores das equipes em saúde. 2007. $80 \mathrm{f}$. Dissertação (Mestrado Profissional de Gestão de tecnologia em Saúde) - Universidade Federal do Rio Grande do Sul, Porto Alegre, 2009.

AZEVEDO, J. C. R.; FAORO, N. T.; XAVIER, E. A. Avaliação de desempenho: um instrumento de gestão e democratização nas relações de trabalho. In: BRASIL. Ministério da Saúde. Secretaria de Gestão do Trabalho na saúde (Org.). Prêmio InovaSUS: valorização de boas práticas e inovação na gestão do trabalho na saúde. 1. ed. Brasília, DF: Ministério da Saúde, 2013. p. 29-35.

BRASIL. Ministério da Saúde. Secretaria de Atenção à Saúde. Política Nacional de Humanização. Contrato interno de gestão: Secretaria Municipal de Saúde Curitiba/PR. Brasília: Ministério da Saúde, 2006.

Ministério da Saúde. Portaria n. 4.279, de 30 de dezembro de 2010. Estabelece diretrizes para a organização da Rede de Atenção à Saúde no âmbito do Sistema Único de Saúde (SUS). Diário Oficial [da] União, Brasília, DF, 31 dez. 2010, Seção 1, p. 88, 2010.

COSTA E SILVA, V. O processo de implantação do Sistema Integrado de Serviços de Saúde em Vitória-ES: contribuição à discussão da integralidade na atenção à saúde. 2004. 151 f. Dissertação (Mestrado em Saúde Pública) - Escola Nacional de Saúde Pública Sergio Arouca, Fundação Oswaldo Cruz, Rio de janeiro, 2004.

Terceiro setor e parcerias na saúde: as

Organizações sociais como possibilidades e limites na gerência da Estratégia Saúde da Família. 2014. 150 f. Tese (Doutorado em Saúde Pública) - Escola Nacional de Saúde Pública Sergio Arouca, Rio de Janeiro, 2014.

\section{COSTA E SILVA, V.; ESCOVAL, A.; HORTALE, V. A.}

Contratualização na Atenção Primária à Saúde: a experiência de Portugal e Brasil. Ciência \&t Saúde Coletiva, Rio de Janeiro, v. 19, n. 8, p. 3593-3604, 2014.

\section{DITTERICH, R. G.; MOYSÉS, S. T.; MOYSÉS, S. J. O} uso de contratos de gestão e incentivos profissionais no setor público da saúde. Cadernos de Saúde Pública, Rio de Janeiro, v. 28, n. 4, p. 615-627, 2012.

DUCCI, L. Curitiba firma contrato de gestão com metas para a saúde. Revista Brasileira Saúde da Família, Brasília, DF, v. 8, n. 14, p. 48-57, 2007.

ESCOVAL, A. Evolução da administração pública da saúde: O papel da contratualização - fatores críticos do contexto português. 2003. 314 f. Tese (Doutorado em Gestão) - Instituto Superior de Ciências do Trabalho e da Empresa, Lisboa, 2003. O processo de contratualização na saúde em Portugal (1996-2005). Revista Portuguesa de Saúde Pública, Lisboa, n. 9, p. 7-24, 2010. 
ESCOVAL, A.; RIBEIRO, R. S.; MATOS, T. T. A contratualização em cuidados de saúde primários: o contexto internacional. Revista Portuguesa de Saúde Pública, Lisboa, n. 9, p. 41-57, 2010.

FORTUNA, C. M. et al. Alguns aspectos do trabalho em saúde: os trabalhadores e os processos de gestão. Saúde em Debate, Londrina, v. 26, n. 62, p. 272-281, 2002.

FREITAS, P.; ESCOVAL, A. A transparência na contratualização em saúde e o papel dos cidadãos. Revista Portuguesa de Saúde Pública, n. 9, p. 129-139, 2010.

MENDES, E. V. Os sistemas de serviços de saúde: o que os gestores deveriam saber sobre essas organizações complexas. Fortaleza: ESP-CE. 2002.

Revisão bibliográfica sobre redes de atenção à saúde. Belo Horizonte: Secretaria de Estado de Saúde de Minas Gerais, 2007.
NEY, M. S.; PIERANTONI, C. R.; LAPÃO, L. V.

Sistemas de avaliação profissional e contratualização da gestão na Atenção Primária à Saúde em Portugal. Saúde em Debate, Rio de Janeiro, v. 39, n. 104, p. 43-55, 2015 .

WORLD BANK. Brazil Enhancing Performance in Brazil's Health Sector: lessons from innovations in the state of São Paulo and the city of Curitiba. Washington, DC: The World Bank, 2006.

Recebido para publicação em maio de 2015

Versão final em outubro de 2015

Conflito de interesses: inexistente

Suporte financeiro: não houve 